\title{
A!
}

This is an electronic reprint of the original article.

This reprint may differ from the original in pagination and typographic detail.

Peltonen, Antti; Nguyen, Hung Q.; Muhonen, Juha T.; Pekola, Jukka P.

\section{Milling a silicon nitride membrane by focused ion beam}

Published in:

JOURNAL OF VACUUM SCIENCE AND TECHNOLOGY. B, NANOTECHNOLOGY \& MICROELECTRONICS

DOI:

$10.1116 / 1.4963895$

Published: 01/11/2016

Document Version

Publisher's PDF, also known as Version of record

Please cite the original version:

Peltonen, A., Nguyen, H. Q., Muhonen, J. T., \& Pekola, J. P. (2016). Milling a silicon nitride membrane by focused ion beam. JOURNAL OF VACUUM'SCIENCE AND TECHNOLOGY. B, NANOTECHNOLOGY \& MICROELECTRONICS, 34(6), 1-6. [062201]. https://doi.org/10.1116/1.4963895

This material is protected by copyright and other intellectual property rights, and duplication or sale of all or part of any of the repository collections is not permitted, except that material may be duplicated by you for your research use or educational purposes in electronic or print form. You must obtain permission for any other use. Electronic or print copies may not be offered, whether for sale or otherwise to anyone who is not an authorised user. 


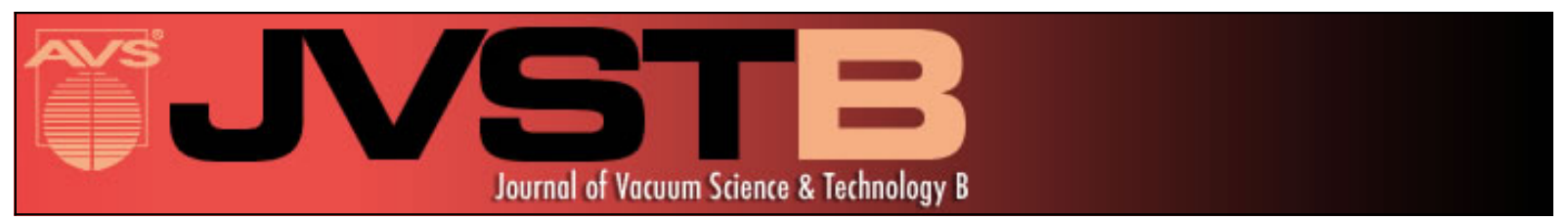

\section{Milling a silicon nitride membrane by focused ion beam}

Antti Peltonen, Hung Q. Nguyen, Juha T. Muhonen, and Jukka P. Pekola

Citation: Journal of Vacuum Science \& Technology B 34, 062201 (2016); doi: 10.1116/1.4963895

View online: http://dx.doi.org/10.1116/1.4963895

View Table of Contents: http://scitation.aip.org/content/avs/journal/jvstb/34/6?ver=pdfcov

Published by the AVS: Science \& Technology of Materials, Interfaces, and Processing

\section{Articles you may be interested in}

Effects of $\mathrm{Ga}+$ milling on InGaAsP quantum well laser with mirrors milled by focused ion beam

J. Vac. Sci. Technol. B 27, L25 (2009); 10.1116/1.3207741

Comment on "Effects of focused ion beam milling on the nanomechanical behavior of a molybdenum-alloy single crystal" Appl. Phys. Lett.91, 111915 (2007)

Appl. Phys. Lett. 92, 096101 (2008); 10.1063/1.2889997

Effects of focused ion beam milling on the nanomechanical behavior of a molybdenum-alloy single crystal Appl. Phys. Lett. 91, 111915 (2007); 10.1063/1.2784948

Controlled fabrication of nickel perpendicular nanocontacts using focused ion beam milling

J. Appl. Phys. 99, 08C501 (2006); 10.1063/1.2150389

Lateral templating of self-organized ripple morphologies during focused ion beam milling of $\mathrm{Ge}$

J. Vac. Sci. Technol. B 23, 1068 (2005); 10.1116/1.1897711

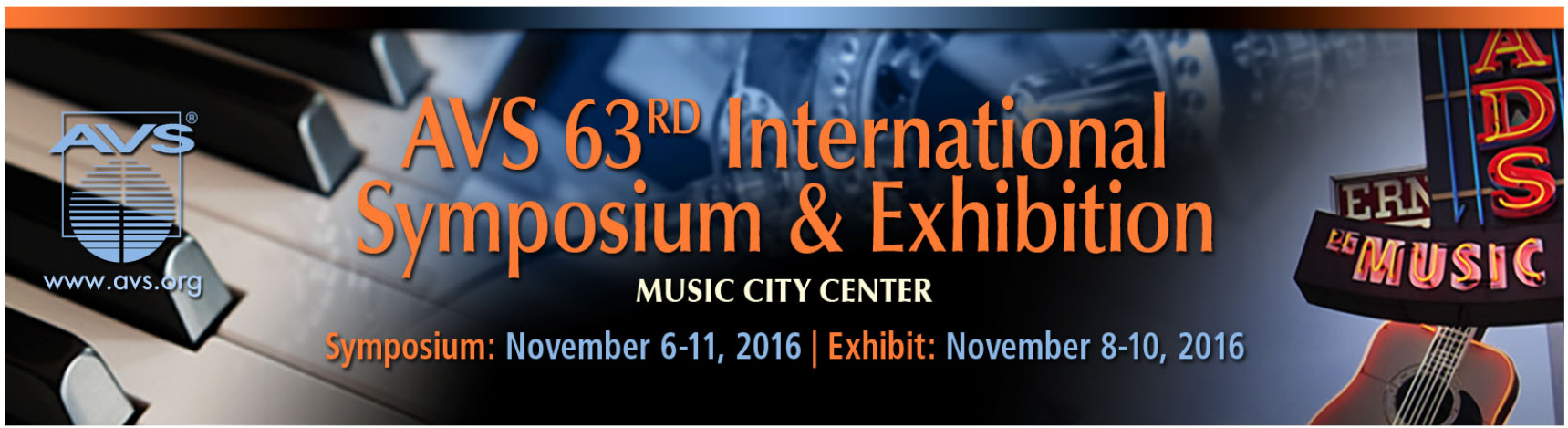




\title{
Milling a silicon nitride membrane by focused ion beam
}

\author{
Antti Peltonen ${ }^{\text {a) }}$ \\ Aalto-NanoFab, Micronova, Aalto University, P.O. 13500, FI-00076 Aalto, Finland \\ Hung Q. Nguyen, Juha T. Muhonen, and Jukka P. Pekola \\ Low Temperature Laboratory, Department of Applied Physics, Aalto University School of Science, \\ FI-00076 Aalto, Finland
}

(Received 20 July 2016; accepted 15 September 2016; published 17 October 2016)

\begin{abstract}
An ultrathin amorphous membrane, such as that made of silicon nitride ( $\mathrm{SiN})$ suspended on silicon substrate, is a popular platform for various applications. However, its hardness causes many difficult technical problems for patterning, especially when combined with other fabrication processes. In nanofabrication, focused ion beam (FIB) is a popular milling technique. It would be a perfect tool for perforating the SiN membrane, but the ion beam charges the membrane, induces stress, and breaks them sporadically. The authors design a metallic structure near the cutting area to neutralize the charges. It reduces stress on the membrane and enables the perforation. Commercial $\mathrm{SiN}$ membranes are perforated with FIB and are suspended on thin legs on silicon chip. The authors study and discuss various designs and the applicability of this approach. (C) 2016 American Vacuum Society. [http://dx.doi.org/10.1116/1.4963895]
\end{abstract}

\section{INTRODUCTION}

Thin film membranes made of amorphous materials are popular in mesoscopic physics and nanotechnology as they provide a two dimensional platform for various experiments and applications. For example, in biological research, silicon nitride membranes are being used as supporting structures in biomedical applications, ${ }^{1,2}$ as a porous mass transfer platform in cell culturing, ${ }^{3}$ as a nanosieve ${ }^{4}$ in fluid separation membranes, or as electrodes in electrochemistry. ${ }^{5}$ In physics applications, nitride membranes are used in photonic nanocrystals ${ }^{6}$ $(\mathrm{PhC})$, in two-dimensional phononic crystal $(\mathrm{PnC})$ structures, $^{7}$ or as a window for $\mathrm{x}$-ray and transmission electron imaging. Of particular interest, silicon nitride membranes are used as an isolated platform ${ }^{8-12}$ that separates cold and hot parts of a thermal machine. A refrigerator that operates at ultralow temperature made from superconducting tunnel junctions ${ }^{13-15}$ stays on the bulk Si and extends its cold fingers to the membrane. To ensure an efficient performance of the refrigerator, the membrane is perforated so that its central piece, the cold part, is suspended on thin legs. It is hard to pattern such fragile membrane and make it compatible with other fabrication steps.

A wide range of amorphous materials and composites can be suspended on a silicon wafer with thickness down to a few tens of nanometer and area up to $\mathrm{mm}^{2}$. However, because they are so inert and durable, it is tricky to pattern them into desired structures. Typically, etching of the membranes is done with highly corrosive chemicals, or performed under extreme physical conditions, such as high temperature and detrimental plasma. Such processes are harmful to the device of interest and are incompatible with other fabrication processes. Moreover, the membranes, now suspended on thin legs, are very fragile and it is nearly impossible to further manipulate them. It is important to develop a perforation method that gives a high yield and is compatible with a wide range of standard microfabrication techniques.

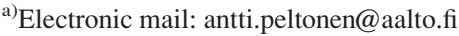

Alternatively, a maskless process can be employed to perforate dielectric membranes. An electron beam at a high energy $^{16,17}$ as well as an helium ion beam ${ }^{18}$ have been used to punch nanopores in $\mathrm{SiN}$ membrane in biophysical research. Here, a high energy beam of accelerated particles sputter material from the target area. In nanofabrication, focused ion beam (FIB) using gallium is well known as a machining tool for modifying devices at micro- and nanometer scales. ${ }^{19-21}$ FIB milling is a slow process, because a small size focused beam sweeps over an area smaller than $1 \mathrm{~mm}^{2}$ at a time, but can very well be used in small batch fabrication, especially in research. The advantage of the FIB milling is that it can easily be done at any stage of the device fabrication due to its local material removal, and absence of chemicals, liquid surface tensions, and thermal stress. FIB has open substrate requirements and can pattern nanometer sized areas with precise alignment. The only requirements for FIB processing are the vacuum compatibility, a low minimum electrical conductivity of the substrate, and that the material hardness does not exceed 9 in Mohs scale. Depending on the application, redeposition of gallium during implantation and milling may affect the target quality. Also, implantation of gallium during the milling cannot be avoided and that might change the target properties such as etch rate. $^{22}$ High energy gallium ions also amorphize a thin surface layer of crystalline target material on a hit, ${ }^{23}$ which is undesirable mostly for HR-TEM imaging. On an insulating surface, such as the suspended SiN membrane, the ion current charges the dielectric membrane, ${ }^{24}$ increases tension, and can break the membrane.

In this paper, we engineer a mesh of metal surrounding the perforated area to neutralize the charged membrane during milling with the FIB [Fig. 1(a)], thus effectively preventing charging. The neutralization mesh is designed so that when milling in specific order, the residual $\operatorname{stress}^{25}$ in the membrane is minimized and thus reduces the possibility of breaking them. The SiN membrane is perforated so that the 
(a)

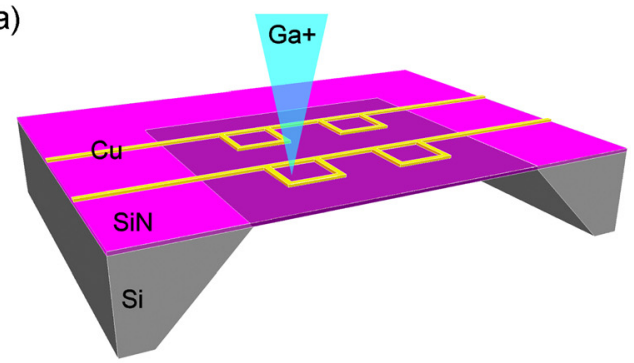

(b)
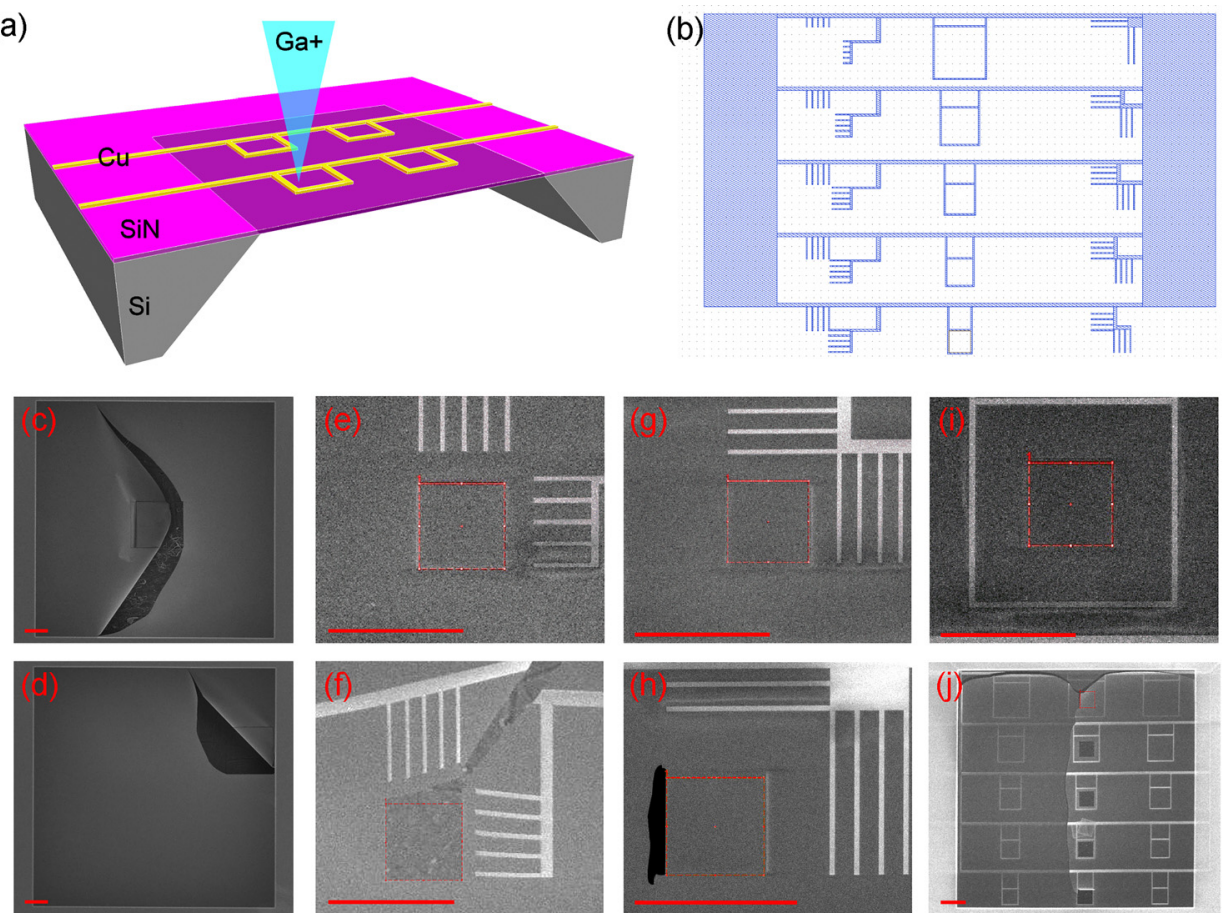

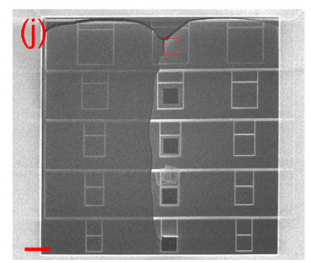

FIG. 1. (Color online) (a) 3D illustration of the experiment. A focused gallium ion beam irradiates the insulating SiN membrane. The perforated area is surrounded with $30 \mathrm{~nm}$ thick copper wires to prevent the buildup of positive charges from the $\mathrm{Ga}^{+}$ions. (b) Layout of the first experiment: Each chip composes of 15 different conductive structures to examine the charge removal. We refer to these structures as copper mesh. Comb shaped patterns which are perpendicular (left) and parallel (right) to the milled area and a square shape (center). The smallest wire width is $4 \mu \mathrm{m}$. The charge conducting wires are grounded to a large area $200 \times 800 \mu \mathrm{m}^{2}$ pads for efficient charge removal from the local area. (c) SEM micrograph of a whole $1 \times 1$ mm ${ }^{2}$ SiN membrane completely covered with $10 \mathrm{~nm}$ platinum layer. The membrane is broken during milling of a $200 \times 200 \mu \mathrm{m}^{2}$ area in the center. (d) A similar cut on a $10 \mathrm{~nm}$ thick platinum layer covered SiN membrane at the corner yield a broken membrane. (e) Milling a membrane with perpendicular comb pattern. A slight change in contrast surrounding the copper wire implies charge buildup near the cutting area (square box). (f) A failed perforation with milling $10 \mu \mathrm{m}$ away from the $\mathrm{Cu}$ wire. (g) and (h) Similar experiment for parallel comb structure, which yields a cracked membrane. Charge buildup can be observed surrounding some of the copper wires. (i) Charge buildup on bottom copper wire during milling one of the largest rectangles. (j) The membrane is broken during milling the largest top most rectangle. The length of the scale bars is $100 \mu \mathrm{m}$.

final outcome is a small center piece that is suspended on thin legs. We study in detail the perforating conditions and discuss the application of this approach.

It would be also possible to remove charging by covering the whole membrane with metal, but metal on the back side of the membrane would have detrimental effect on sensitive devices on the top surface. The top side is not possible to cover with extra material layers, since it contains devices. It is also necessary to avoid increasing device fabrication time, by minimizing the amount of surface area that should be covered by electron beam lithography exposure by not exposing any unnecessary patterns in the process step. Drawing narrow patterns is a better choice than covering large areas in electron beam lithography. In our case, the mesh itself is possible to fabricate in the same lithography process steps as the functional devices on the device platform that need environmental isolation, as they both can be fabricated of the same $30 \mathrm{~nm}$ thick copper. Fabricating both, the mesh and devices, in the same process steps saves time, reduces cost, and keeps the whole process simpler.

\section{FABRICATION}

All silicon chips are diced into $5 \times 5 \mathrm{~mm}^{2}$ squares, with a suspended $1 \mathrm{~mm} \times 1 \mathrm{~mm} \times 100 \mathrm{~nm}$ SiN-membrane in the middle. They are either ordered from Norcada ${ }^{26}$ or made in- house. Poly(methyl methacrylate) of $200 \mathrm{~nm}$ from MicroChem was spun at 2500 round per minute for $1 \mathrm{~min}$, and then baked at $170^{\circ} \mathrm{C}$ for $5 \mathrm{~min}$. Charge neutralization patterns were exposed by VISTEC EBPG 5000+ES electron beam lithography system with current $50 \mathrm{nA}$ and dose $1000 \mu \mathrm{C} / \mathrm{cm}^{2}$. The sample is developed in 1:3 solution of methyl isobutyl ketone and isopropanol for $1 \mathrm{~min}$. It is then loaded in a Telemark 241 electron beam evaporator, and $30 \mathrm{~nm}$ of copper is deposited on top. Lift-off of $\mathrm{Cu}$ was done at temperature $80^{\circ} \mathrm{C}$ in mr-Rem 400 or mr-Rem 500 from Microresist for $10 \mathrm{~min}$, rinsed in isopropanol, and dried with dry nitrogen air flow.

Test membranes were loaded into a FEI Company (Helios Nanolab 600) focused dual beam system and perforated with gallium ions at $30 \mathrm{kV}$. Electron micrographs are taken in situ during the perforation.

\section{EXPERIMENT}

An initial study of thin $100 \mathrm{~nm} \mathrm{SiN} \mathrm{membranes} \mathrm{proved}$ that a low current of $0.92 \mathrm{nA}$ on a nonpatterned membrane is sufficient to very often cause the membrane to over charge and crack. Milling with low currents does not provide sufficient protection against charging and they increase milling speeds significantly. Repeated exposure of these low current beams over the same pattern, therefore, cannot be used to 
perforate the membrane successfully and within acceptable speed. On the other hand, membranes completely covered with thin layer of metal reduces cracking rate significantly. Also, it was noted that currents higher than $2.8 \mathrm{nA}$ can break the membrane, even with some charge conduction present [Figs. 1(c) and 1(d)]. Based on these facts, two studies were carried out to investigate how to reduce the residual stress on the membrane, and speed up the milling, without having to cover the whole membrane in the metal layer. In the first experiment, we want to find out a suitable perforation mesh shape [Fig. 1(b)]: a comb shape [Figs. 1(e)-1(h)] or a square shape [Figs. 1(i) and 1(j)]. We change the distance between the comb shape structure and the milling area and observe when the membrane breaks. A second study focuses on cutting near a square shape [Fig. 1(j)]. Distances between the perforated region and the conducting wire were varied and statistics of membrane breakage were collected. A total of 29 sequences for parallel comb and 30 sequences for perpendicular combs were cut for the experiment. An ion beam of $2.8 \mathrm{nA}$ was used in cutting and was chosen as a compromise between the cutting speed and avoiding heavy local charging. Choosing a $2.8 \mathrm{nA}$ ion current was considered justified, and due to the device limitations and specifications constrained by the given device, we needed to fabricate on the device platform. These devices on the device platform are for other experiments and lie outside of the scope of this paper. By taking the electron image in situ while cutting, it is possible to observe the behavior and the buckling of the membrane and to determine if the membrane perforation was either successful, or not. The buckling itself is difficult to observe in action with SEM, but can also be confirmed by observing the failing adhesion and wrinkles appearing into deposited metal wires after heavy charging of the membrane.

For testing the efficiency of the separation distance, rectangle shaped conductive patterns were used because they performed the best compared to comb shapes. A total of 50 sequences were used where each membrane was patterned with copper rectangles of sizes $60,70,80,100$, and $140 \mu \mathrm{m}$ [Fig. 2(a)]. A square milling shape of size $60 \times 60 \mu \mathrm{m}^{2}$ [squares in Figs. 1(e)-1(j) and 2(a)] was milled with the FIB at the center of the each pattern, starting the milling from the smallest square. Selected design generates five different separation distances to be tested in the test membranes. The separation distance in the smallest square pattern is $0 \mu \mathrm{m}$, and then increases to $5,10,20$, and $40 \mu \mathrm{m}$ at the largest square. After milling the smallest square, and if the membrane did not break, the milling advances to next largest square. The whole sequences of different size square patterns are milled from smallest to largest using the same milling area. If the membrane did not break at the largest separation distance of $40 \mu \mathrm{m}$, we mark the membrane cut as successful, as it survived all five cuts with different separation distances. Each membrane had three columns of five different sized squares. This makes it possible to run at most three complete (a)
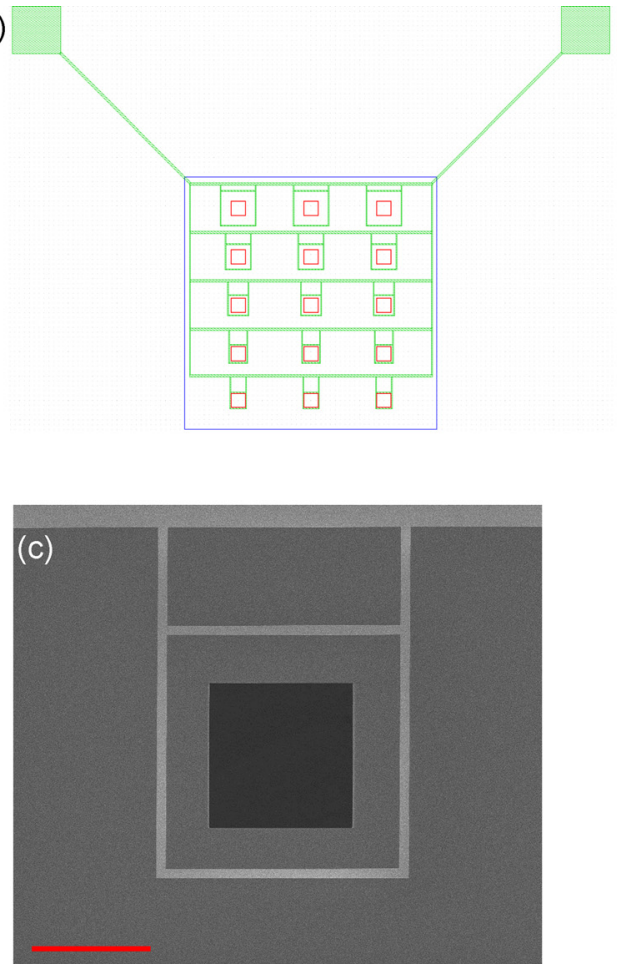
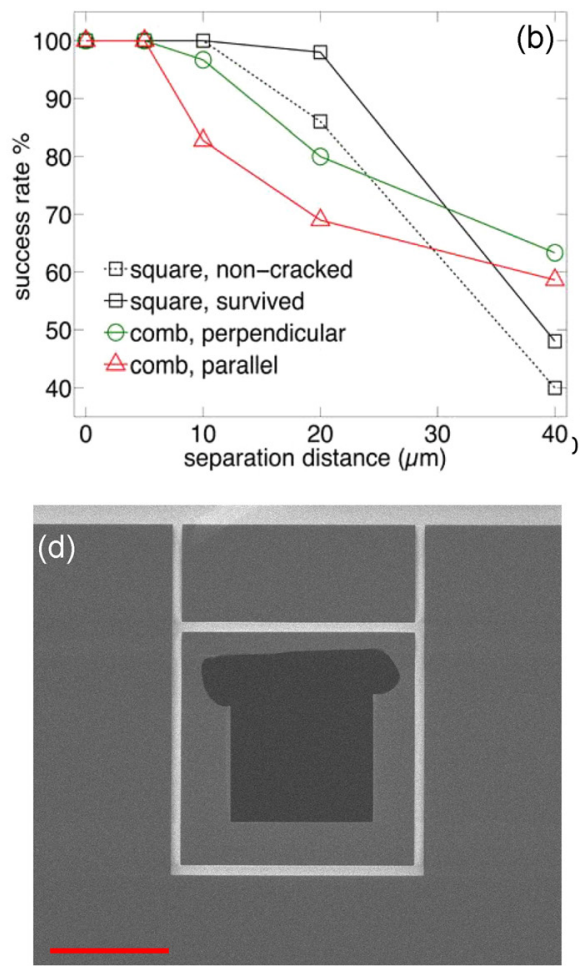

FIG. 2. (Color online) (a) Layout of the square test pattern. Silicon nitride window of $1 \times 1 \mathrm{~mm}^{2}$ (large rectangle) is patterned with $30 \mathrm{~nm}$ thick copper (striped area). The patterned copper composes of 15 test squares for experimentation. The ion beam is used to perforate a square of $60 \times 60 \mu \mathrm{m}^{2}$ (small squares) in the center of each $\mathrm{Cu}$ square. Two Cu pads size of $200 \times 200 \mu \mathrm{m}^{2}, 750 \mu \mathrm{m}$ away from the SiN window, aim at anticharging. (b) Main result: perforation success rate as a function of separation distance. We distinguish the two kinds of damages: cracked membranes and membranes totally destroyed. Results of nonbroken perpendicular and parallel comb pattern millings are also shown. (c) A successful cut with $20 \mu$ m separation distance. (d) An unsuccessful cut with $20 \mu$ m separation distance. The damage is a result of membrane charging and buckling, which might result from not having enough conductive material in the vicinity of the milling area. The length of the scale bars is $50 \mu \mathrm{m}$. 
sequences on each membrane. If the membrane survives the first sequence, the second sequence is run on the same membrane, and if the membrane survives then the third sequence is run, until all of the 15 squares have been perforated.

After confirming the most effective conductive pattern for the application, we turn our focus on applying this knowledge into practical solution. The original problem was to isolate a thermal device from the environment by perforating the thin SiN membrane into the suspended platform. We pattern a $30 \mathrm{~nm}$ thick copper mesh [Fig. 3(a)] together with our thermal devices on the $\mathrm{SiN}$ membrane, and use this mesh as charge conductive pattern and as a guidelines for cutting. After the platform has been released from the bulk silicon, all of the copper mesh patterns and SiN residue should be removed as milling debris by then leaving no unnecessary material around [Fig. 3(c)]. The released device platform with thermal devices on it is then used for further experiments, not reported in this paper.

\section{RESULTS}

To find a suitable conducting pattern for discharging, three different patterns were selected for testing, two comb shapes and a square [see Fig. 1(b)]. After cutting several membranes, we noticed that the membranes patterned with parallel comb break most often [Figs. 1(g), 1(h), and 2(b)]. Strongest charging halo was observed in this pattern type. It is possible that the spike of the comb nearest to milling area has to remove most of the charge, but cannot do it properly alone. Thicker and wider spikes might improve the perforation. Statistics for cutting the comb structure is shown in Fig. 2(b). Least charging effects were observed in the square pattern. The square pattern surrounds the whole milled area and thus effectively collects all charge applied to the area. The fact that we can perforate the membrane with a neutralization structure confirms our assumption that it is the charging from the gallium ions that induce stress and cause the membrane to break. The rectangular pattern was selected for further testing, since it appeared to be the most promising.
After selecting the pattern design, we then focus on perforating squares of $60 \times 60 \mu \mathrm{m}^{2}$ dimension. This size is a good trade-off between cutting speed and yield. Cutting a hollow square of this size on $100 \mathrm{~nm}$ thick SiN membranes takes 53 $\mathrm{s}$ with a $2.8 \mathrm{nA}$ beam current, which is a reasonable speed. By cutting a larger area, the perforation gets slightly faster, but then the breaking rate of the membranes rapidly increases due to uneven stress produced on the membrane. From the experience of processing several full size membranes, it is safe to cut a square hole of $100 \times 100 \mu \mathrm{m}^{2}$ or smaller without any stress reduction. For the experiment, we stick to the $60 \times 60 \mu \mathrm{m}^{2}$ square. This perforation size can be milled at any location on the membrane, without increasing considerably the probability of breaking it. The maximum hole size on the membrane without a strain stress reduction is a square of size $200 \times 200 \mu \mathrm{m}^{2}$. In a hole size of this the charge neutralization pattern becomes irrelevant, since now it is the strain stress that starts to dominate the membrane breaking mechanism. This could be confirmed by painting the whole membrane with a $10 \mathrm{~nm}$ platinum layer and trying to cut a large hole in it in various locations with catastrophic consequences [Figs. 1(c) and 1(d)]. Perforating a single hole of this size or larger turns out to be not possible at all.

Out of 50 test sequences with square patterns, all survived the separation distances of 0,5 , and $10 \mu \mathrm{m}$ without problems. Problems start to accumulate when distance is $20 \mu \mathrm{m}$ or higher. Membranes that are not cracked at all are considered survived membrane. Membranes that are slightly crack are labeled as cracked. At $20 \mu \mathrm{m}$ distance, six out of 50 is cracked, from which one is completely broken, thus yielding a $12 \%$ crack rate and a $98 \%$ success rate. At $40 \mu \mathrm{m}$, numbers get even higher, and $60 \%$ of the membranes are cracked and $52 \%$ are broken totally [Fig. 2(b)]. The charging halo on the membrane became most visible at the patterns that had 20 and $40 \mu \mathrm{m}$ separation distance to the $\mathrm{Cu}$ neutralization wire, and these were the same patterns that were involved with the most membrane breaking [Figs. 2(b) and 2(d)] as shown in the result summary chart. Apparently, a distance of $10 \mu \mathrm{m}$
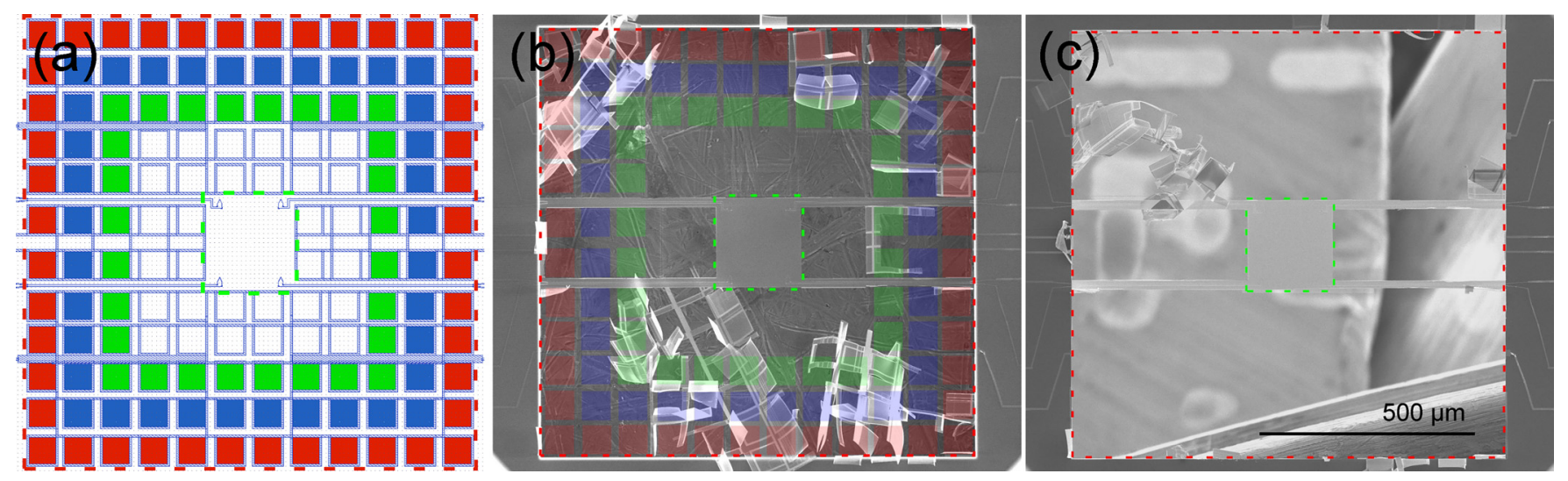

FIG. 3. (Color online) (a) Layout of $30 \mathrm{~nm}$ thick copper mesh structure for device platform release milling. The three layers of solid squares define the cutting sequence. (b) A false-color SEM image of strain stress reducing cutting strategy for a full size $1 \times 1 \mathrm{~mm}^{2} 100 \mathrm{~nm}$ thick SiN membrane. (c) A successful perforation with help of the $\mathrm{Cu}$ mesh. The smaller dashed line rectangle marks the device platform that is suspended on $20 \mu \mathrm{m}$ wide bridges. The larger dashed line rectangle indicates the edge of the $1 \mathrm{~mm}$ window. The background in the image is manipulated darker so that the electron transparent membrane on the front is more visible. Very often, the perforated material remains attached to the membrane, but most of it is removed when the FIB chamber is vented to atmospheric pressure. The SiN debris from milling the mesh can be seen in (b) and (c). 
from the conductor provides an efficient charge cancellation for square patterns and thus guarantees a successful milling of the membrane. For comb patterns, the safe distance is lower and starts from $5 \mu \mathrm{m}$. This is our most important result. It clearly demonstrates that the guidance of the produced charging is necessary when perforating a nonconducting film, and out of the three different tested pattern types, the rectangle works the best. Furthermore, we demonstrated that the effect is distance dependent.

Perforated membranes and membranes containing strain stress are both fragile. If membranes under strain stress have to be perforated, it is desirable to perforate them into a shape that gives up in order to reduce or relax the stress. To perforate the whole membrane into an shape that gives up, e.g., spider web, suspended bridges, zig zag, spiral, or serpentine shape, it is better to start cutting not larger than $100 \mu \mathrm{m}$ squares at opposite sides from the edge. In order to perforate and relax our test membrane, we pattern a $30 \mathrm{~nm}$ thick copper mesh on the area (Fig. 3). The membrane is relaxed by cutting first the outermost layer of squares [red squares in Figs. 3(a) and 3(b)] around the edge, and then proceeding toward the membrane center with the next layer (blue squares, and then green squares). Cutting is best done evenly from opposing sides, few squares at the time, to prevent imbalanced stress build up, which could break the membrane. This way the stress load is reduced evenly, leaving the isolated device platform in the center [Fig. 3(c)]. Cutting too much at once from one side or from the center of the membrane significantly increases the risk of breaking it. This happens because the imbalance of stress between opposing edges will tear the membrane. In this experiment, $60 \times 60 \mu \mathrm{m}^{2}$ size squares were cut around the membrane edge, no more than three squares at a time on opposing edges. A square hole of $60 \times 60 \mu \mathrm{m}^{2}$ was considered to be small enough to have some extra safety margin compared to $100 \times 100 \mu \mathrm{m}^{2}$ size hole, thus making sure not to cut too large holes. Once three circles of rectangles were milled around the edge, so that the device platform was surrounded by a flexible web, we cut the remaining mesh area in random order. Final stage was that all holes were connected so that an isolated island with devices was left hanging from a few narrow bridges [Fig. 3(c)].

\section{DISCUSSION}

Having an excellent mechanical and chemical durability, silicon nitride is the most common membrane available for research and applications in microfabrication. However, with a typical thickness less than a micron-an ultrahigh aspect ratio, this amorphous membrane is extremely fragile to external impacts. The nitride film stress phenomenon with a yield stress in the order of $1 \mathrm{GPa}$ is well known to exist, especially between the silicon substrate and silicon nitride thin film, ${ }^{27}$ where a thin silicon oxide film is used between the two in device fabrication to reduce detrimental stress. ${ }^{25}$ Normally, the stress is highest at the boundary, where the bulk substrate terminates. A high and uneven load on the membrane tends to break it. Compared to the silicon substrate, the membrane is difficult to handle without damaging it, for example, at the phase boundary of liquid and vapor, a common step for any process. Typically, the membrane perforation is left as the final step to avoid further manipulation. Applied to a local area, and without high temperature or corrosive plasma, FIB is the safest choice, especially when the membrane contains other devices from previous fabrication steps.

When using FIB, the main cause for the breaking of membranes arises from the accumulation of charges on such nonconducting surfaces. This is typically visible as a halo around the surrounding conductor in the cutting area. This difference in contrast in the electron micrographs lasts only a couple of seconds and is difficult to capture. Still, it is a precursor of a failure of a cut. The less conducting the surface, the more visible the halo is, and thus more violent the breaking of the membrane. Such halo's or breakings are not present in membranes completely coated with thin layer of metal. Depending on application, it is possible that repeated low exposure can be used to reduce the local charge accumulation, but in this study, that option was not being studied, as our method was considered faster and more reliable for our application.

Perforating the SiN membrane using FIB offers two major advantages. First, milling with the ion beam is a physical process. It sputters any materials, being metal, oxide, nitride, carbide, or composite. This is an important feature, as the ion beam can be used at any step and has no chemical selectivity requirement. Etching inert materials of the membrane requires corrosive etchants or reactive plasma. This places a huge constraint on the fabrication compatibility point of view. In comparison, the only requirement for FIB processing is vacuum compatibility. Second, FIB is a maskless process that directly sputters material according to a customized design. This is especially fit for research where in house experience is highly important. A wide range of shapes and geometries can be milled directly, and the system is not limited to squares. These two features enable the FIB approach to be employed on a membrane of virtually any material and thickness, as well as any shape and size.

FIB can be used in two milling modes, serial and parallel. Experiments done in this work were performed with serial milling due to its easier use in the first experiments. The advantage with parallel milling is that the whole pattern can be drawn at once, which might help in avoiding the stress reduction phase. If all patterns are being perforated simultaneously on the whole nitride window scale, that might avoid the stress build-up in any location. The remaining problems in the parallel milling are the possible drift during long milling sessions which could dislocate the milling patterns and the limited resolution. Comparing the two milling modes could be a possible future experiment.

\section{SUMMARY}

We reported a method to perforate a dielectric membrane using focused gallium beam. The charging due to the ion beam is neutralized with $30 \mathrm{~nm}$ thick $\mathrm{Cu}$ structures in the 
vicinity of the area to be perforated. We found that a square box surrounding the cutting area yields the best result. Furthermore, cutting within a distance of $10 \mu \mathrm{m}$ from the metal wire connected to charge depletion area allows successful milling of the membrane. With this approach, we were able to perforate a whole membrane with $20 \mu \mathrm{m}$ wide thin legs on the device platform. Our approach is compatible with a wide range of processes in nanofabrication.

\section{ACKNOWLEDGMENTS}

The authors appreciate the support of the Otanano Otaniemi Research Infrastructure for Micro and Nano Technologies. Samples were fabricated in the Micronova Nanofabrication Center of Aalto University.

${ }^{1}$ S. Zhang et al., Biomaterials 29, 3993 (2008).

${ }^{2}$ I. Vlassiouk, P. Y. Apel, S. N. Dmitriev, K. Healy, and Z. S. Siwy, Proc. Natl. Acad. Sci. U. S. A. 106, 21039 (2009).

${ }^{3}$ M. Ni, W. H. Tong, D. Choudhury, N. A. A. Rahim, C. Iliescu, and H. Yu, Int. J. Mol. Sci. 10, 5411 (2009).

${ }^{4}$ H. D. Tong, H. V. Jansen, V. J. Gadgil, C. G. Bostan, E. Berenschot, C. J. M. van Rijn, and M. Elwenspoek, Nano Lett. 4, 283 (2004).

${ }^{5}$ Y. H. Lanyon, G. De Marzi, Y. E. Watson, A. J. Quinn, J. P. Gleeson, G. Redmond, and D. W. M. Arrigan, Anal. Chem. 79, 3048 (2007).

${ }^{6}$ T. Stomeo, A. Qualtieri, F. Pisanello, L. Martiradonna, P. P. Pompa, M. Grande, D'Orazio, and M. De Vittorio, Advances in Photonic Crystals (InTech, 2013), p. 221.

${ }^{7}$ N. Zen, T. A. Puurtinen, T. J. Isotalo, S. Chaudhuri, and I. J. Maasilta, Nat. Commun. 5, 3435 (2014).

${ }^{8}$ M. M. Leivo and J. P. Pekola, Appl. Phys. Lett. 72, 1305 (1998).
${ }^{9}$ A. Luukanen, M. M. Leivo, J. K. Suoknuuti, A. J. Manninen, and J. P. Pekola, J. Low Temp. Phys. 120, 281 (2000).

${ }^{10}$ A. M. Clark, N. A. Miller, A. Williams, S. T. Ruggiero, G. C. Hilton, L. R. Vale, J. A. Beall, K. D. Irwin, and J. N. Ullom, Appl. Phys. Lett. 86, 173508 (2005).

${ }^{11}$ N. A. Miller, G. C. O’Neill, J. A. Beall, G. C. Hilton, K. D. Irwin, D. R. Schmidt, L. R. Vale, and J. N. Ullom, Appl. Phys. Lett. 92, 163501 (2008).

${ }^{12}$ V. Yefremenko et al., Appl. Phys. Lett. 94, 183504 (2009).

${ }^{13}$ J. T. Muhonen, M. Meschke, and J. P. Pekola, Rep. Prog. Phys. 75, 046501 (2012).

${ }^{14}$ F. Giazotto, T. T. Heikkilä, A. Luukanen, A. M. Savin, and J. P. Pekola, Rev. Mod. Phys. 78, 217 (2006).

${ }^{15}$ M. Nahum, T. M. Eiles, and J. M. Martinis, Appl. Phys. Lett. 65, 3123 (1994).

${ }^{16}$ D. G. Howitt, S. J. Chen, B. C. Gierhart, R. L. Smith, and S. D. Collins, J. Appl. Phys. 103, 024310 (2008).

${ }^{17}$ A. J. Storm, J. H. Chen, X. S. Ling, H. W. Zandbergen, and C. Dekker, Nat. Mater. 2, 537 (2003).

${ }^{18}$ M. M. Marshall, J. Yang, and A. R. Hall, Scanning 34, 101 (2012).

${ }^{19}$ FIB Nanostructures, edited by Z. M. Wang (Springer, New York, 2013).

${ }^{20}$ C. Duan, W. Wang, and Q. Xie, Biomicrofluidics 7, 026501 (2013).

${ }^{21}$ Y. Nakashima and T. Yasuda, Sens. Actuator, A 139, 252 (2007).

${ }^{22}$ N. Chekurov, K. Grigoras, A. Peltonen, S. Franssila, and I. Tittonen, MRS Proc. 1181, DD07-01 (2009).

${ }^{23}$ J. Mayer, L. A. Giannuzzi, T. Kamino, and J. Michael, MRS Bull. 32, 400 (2007).

${ }^{24}$ S. Yogev, J. Levin, M. Molotskii, A. Schwarzman, O. Avayu, and Y. Rosenwaks, J. Appl. Phys. 103, 064107 (2008).

${ }^{25}$ J. Laconte, D. Flandre, and J.-P. Raskin, Micromachined Thin-Film Sensors for SOI-CMOS Co-integration (Springer, Dordrecht, 2006), p. 3.

${ }^{26}$ Norcada Inc., Part number NX5100C, www.norcada.com.

${ }^{27}$ W. Lang, Mater. Sci. Eng. 17, 1 (1996). 